\title{
Transition-Metal-Catalyzed Amination of Aryl Fluorides
}

\author{
Qi-Kai Kang ${ }^{\mathrm{a}, \mathrm{b}}$ \\ Yunzhi Lin ${ }^{a, b}$ \\ Yuntong $\mathbf{L i}^{\mathrm{a}, \mathrm{b}}$ \\ Hang Shi*a,b (iD) \\ a Institute of Natural Sciences, Westlake Institute for \\ Advanced Study, 18 Shilongshan Road, Hangzhou 310024 \\ Zhejiang Province, P. R. of China \\ ${ }^{b}$ Key Laboratory of Precise Synthesis of Functional Molecules \\ of Zhejiang Province, School of Science, Westlake University, \\ 18 Shilongshan Road, Hangzhou 310024, Zhejiang Province, \\ P. R. of China \\ shihang@westlake.edu.cn
}

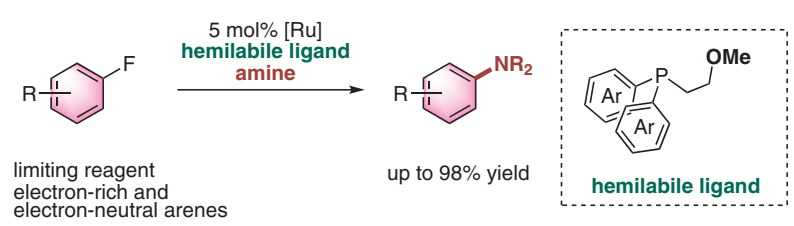

Received: 05.04.2020

Accepted after revision: 19.04.2020

Published online: 14.05 .2020

DOI: 10.1055/s-0040-1707118; Art ID: st-2020-p0193-sp

Abstract Arene activation via transition-metal (TM) $\eta^{6}$-coordination has merged as a powerful method to diversify the aromatic C-F bond, which is relatively less reactive due to its high bond energy. However, this strategy in general requires to use largely excess arenes or $\mathrm{TM} \eta^{6}$ complexes as the substrates. Herein, we highlight our recent work on the catalytic $\mathrm{S}_{\mathrm{N}} \mathrm{Ar}$ amination of electron-rich and electron-neutral aryl fluorides that are inert in classical $\mathrm{S}_{\mathrm{N}} \mathrm{Ar}$ reactions. This protocol enabled by a Ru/hemilabile ligand catalyst covers a broad scope of substrates without wasting arenes. Mechanistic studies revealed that the nucleophilic substitution proceeded on a Ru $\eta^{6}$-arene complex, and the hemilabile ligand significant promoted the arene dissociation.

Key words $\eta^{6}$-complex, hemilabile ligand, amination, $\mathrm{C}-\mathrm{F}$ bond activation, $\mathrm{S}_{\mathrm{N}} \mathrm{Ar}$, ruthenium

Anilines are frequently found as structural components of pharmaceutically relevant compounds, polymers, and natural products. Hitherto, transition-metal-catalyzed aminations of aryl halides, such as Hartwig-Buchwald ${ }^{1}$ reaction and Ullmann reaction ${ }^{2}$, are among the most important accesses to anilines and their derivatives (Scheme 1, a). The reactivity trend of substrates is inversely proportional to dissociation energy of their carbon-halogen bonds. Compared with other halides, aryl fluorides are uncommon coupling partners because of the low reactivity for oxidative addition attributed to the strength of C-F bond, which is the strongest C-heteroatom bonds in nature. In 1973, Kumada and co-workers discovered that a $\mathrm{Ni}(0)$ species served as an efficient catalyst for $\mathrm{C}-\mathrm{C}$ bond formations through cleaving inert $\mathrm{C}-\mathrm{F}$ bonds. ${ }^{3}$ Prompted by this seminal work, in 2013, Wang disclosed a Ni-catalyzed amination of aryl fluorides by using a bulky $N$-heterocyclic carbene ligand (Scheme 1, b). ${ }^{4}$ Various $N$-dialkyl or $N$-alkylaryl secondary amines are compatible under the reaction conditions. In

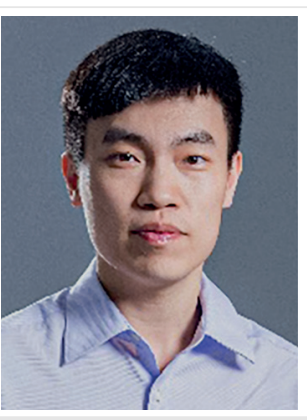

Hang Shi was born and grew up in Liaoning, P. R. of China. His graduate studies were carried out at Peking University under the direction of Prof. Zhen Yang culminating in a PhD degree in 2013. Then, he joined the group of Prof. Tobias Ritter at Harvard University as a postdoctoral researcher. During that period, he also performed part of the research at the Massachusetts General Hospital (MGH). At the end of 2015, he moved to the Scripps Research Institute and joined Prof. Jin-Quan Yu's group. In 2018, he began his independent career at Westlake University, P. R. of China. His research interest focuses on organometallic methodology and asymmetric catalysis.

2018, Sawamura reported a Ni-catalyzed amination with primary amines enabled by a bidentate phosphine ligand, which features a rigid backbone (Scheme 1, c). ${ }^{5}$ Except nickel, silver carbonate was recently addressed as an effective catalyst in the amination of electron-deficient aryl fluorides by Fang (Scheme $1, \mathrm{~d}){ }^{6}$

In addition to the TM redox catalysis, amination of aryl fluorides can also be achieved through a nucleophile addition-elimination process (nucleophilic aromatic substitution, $S_{N} A r$, see Scheme 2, a). ${ }^{7}$ Classical $S_{N} A r$ reactions are limited to electron-deficient aromatics that bear a strong electron-withdrawing group (EWG). Alternatively, instead of a permanent EWG, transition metals such as $\mathrm{Cr}$, Fe, and Mn could also facilitate $S_{N} A r$ reactions via $\eta^{6}$-coordination that enhances the electrophilicity of the ligated aromatic 


\section{$\sigma$-bond activation}

a. Hartwig-Buchwald and Ullmann amination

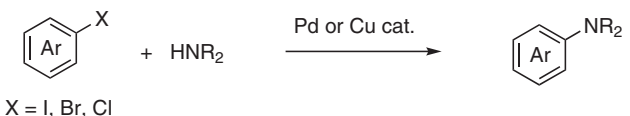

b. Wang (2013)
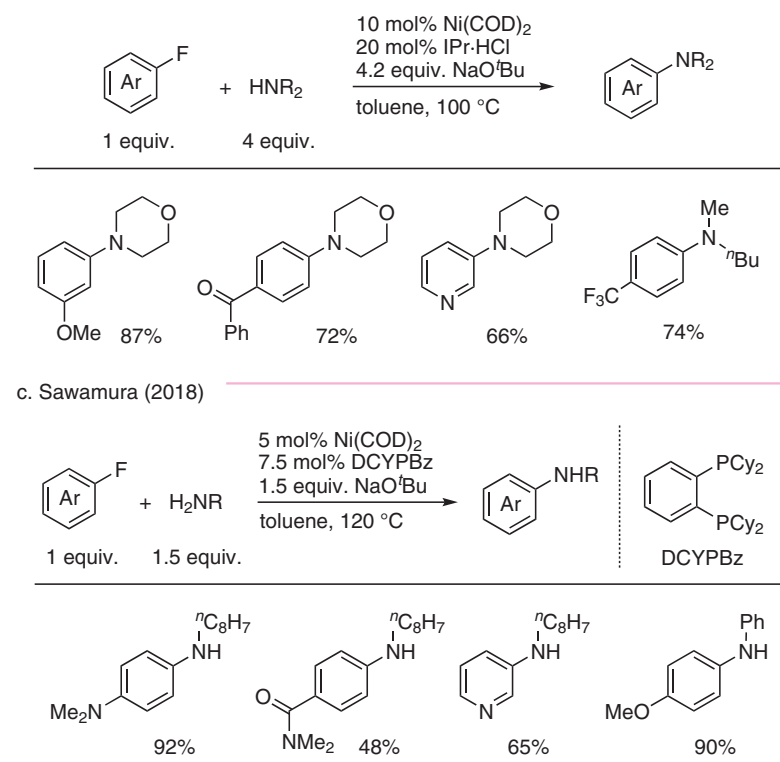

d. Fang (2018)
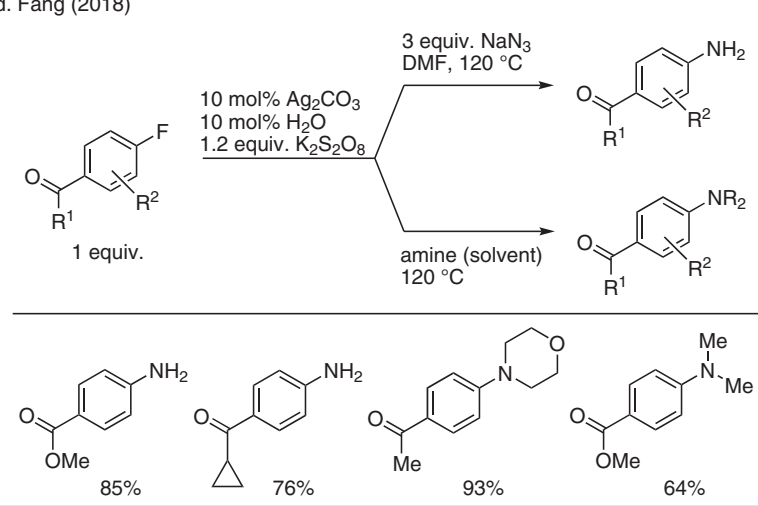

Scheme 1 Amination of aryl fluorides via $\sigma$-bond activation

rings (Scheme 2, b). ${ }^{8}$ Although such reactivity has been explored for more than 60 years after the first chromium $n^{6}$ arene complex reported by Fischer and Hafner ${ }^{9}$, catalytic $\mathrm{S}_{\mathrm{N}} \mathrm{Ar}$ aminations enabled by TM coordination are still rare (Scheme 2, c). ${ }^{10}$ In 2010, Shibata and co-workers reported two Ru-catalyzed aminations of aryl fluorides by using either a tridentate PCP ligand or a monodentate phosphine ligand (Scheme 2, d). ${ }^{10 \mathrm{~d}, \mathrm{e}}$ In both protocols, excess aryl fluorides were required to facilitate arene exchange of $n^{6}$-complexes. The catalytic $\mathrm{S}_{\mathrm{N}} \mathrm{Ar}$ reaction of aryl fluorides as limiting reagents remains to be demonstrated.

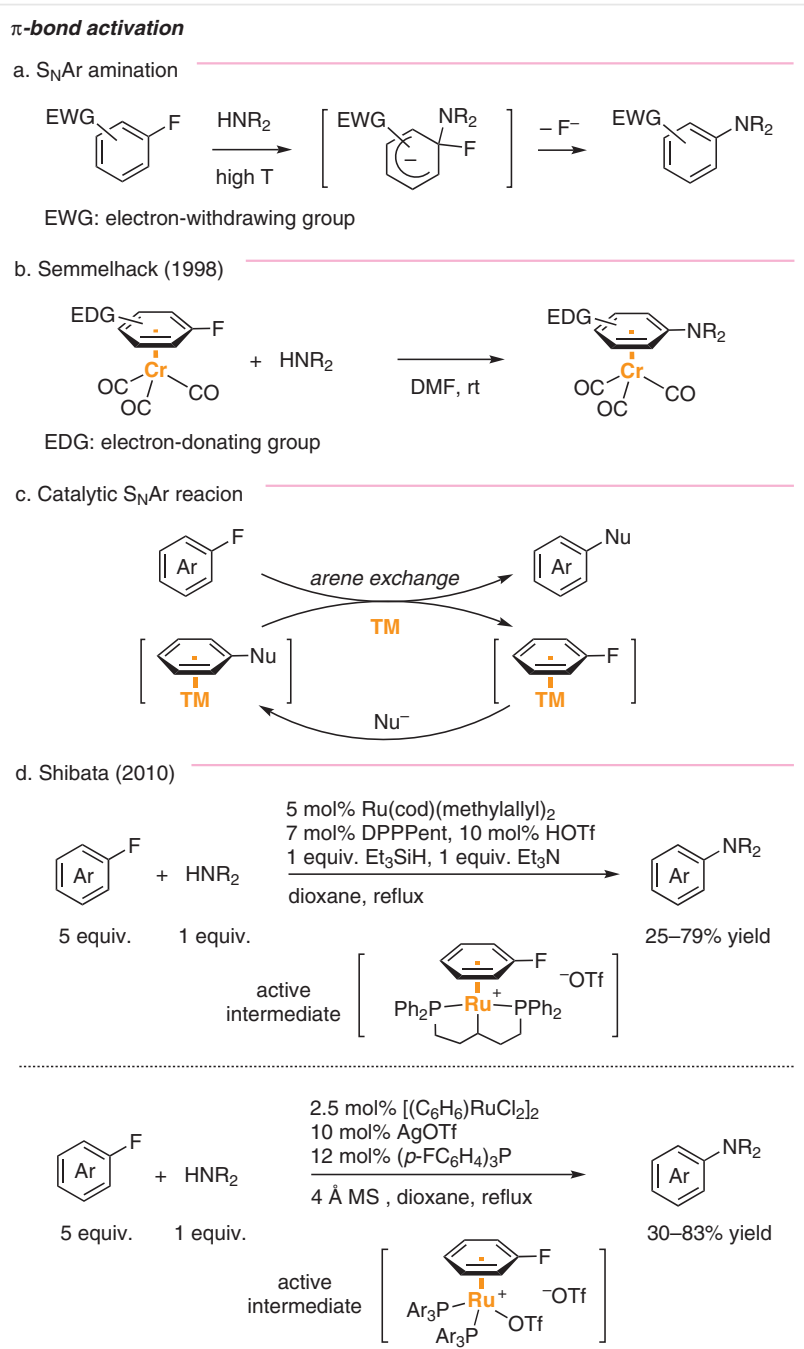

Scheme 2 Amination of aryl fluorides via $\eta^{6}$-coordination

For catalytic arene activations via TM $\eta^{6}$-coordination, the reaction efficiency was markedly affected by substrate association and product dissociation, which referred to as arene exchange. When the metal center maintains the same charge, a typical obstacle in arene exchange is the detachment of one double bond from a relatively stable $\eta^{6}$-complex, generating the corresponding $\eta^{4}$-intermediate (Scheme 3, a). In 1970s, Mahaffy and Pauson found that donor molecules such as THF and cyclohexanone could benefit arene exchange. ${ }^{11}$ Following this observation, several ligands bearing a coordinating side chain were addressed to accelerate the exchange rate. ${ }^{10 g, 12}$ However, examples for the ligand-promoted $\mathrm{S}_{\mathrm{N}} \mathrm{Ar}$ reaction by the strategy outlined above are absent. To overcome the obstacle, we envisioned to utilize hemilabile, bidentate ligands, which could benefit catalytic $\mathrm{S}_{\mathrm{N}} \mathrm{Ar}$ reactions in two ways (Scheme 3, b): a sidechain group L' that coordinates to TM temporarily may promote product dissociation through a steric repulsion; the li- 
gand's hemilabile nature may provide flexibility to stabilize reaction intermediates, as well as reduce steric effects in the coordination of substrates to the catalyst. Herein, we highlight our recent work on the catalytic $S_{N} A r$ amination of electron-rich and electron-neutral aryl fluorides. ${ }^{13}$

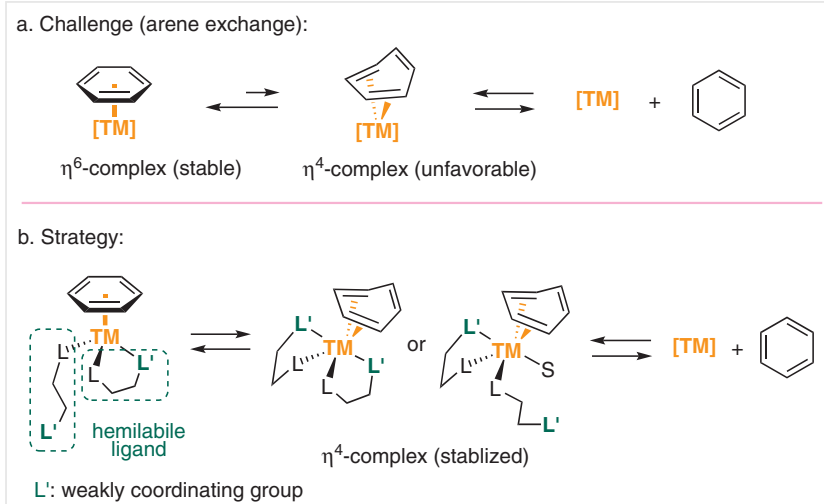

Scheme 3 Arene exchange

We selected fluorobenzene as a limiting reagent to explore the feasibility of our ligand design (Scheme 4). Several representative monodentate phosphine ligands were investigated first, but only a trace amount of desired product was detected. Following our design, when a weakly coordinating group, OMe, was installed on a simple phosphine, a dramatic improvement of yield was obtained (L1, 42\%). We then examined the influence of the linker between the binding atoms $\mathrm{P}$ and $\mathrm{O}$ and found that changing either its flexibility or the steric environment around oxygen reduced the yield

chelating group:

optimal conditions
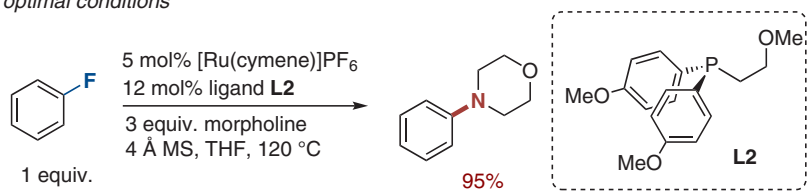

Scheme 4 Catalyst development for $\mathrm{S}_{\mathrm{N}} \mathrm{Ar}$ amination or even inhibited the reaction. Next, an array of other donating groups such as thioether, amine, and phosphine were examined and proved ineffective, demonstrating the importance of matching the two chelating groups on the
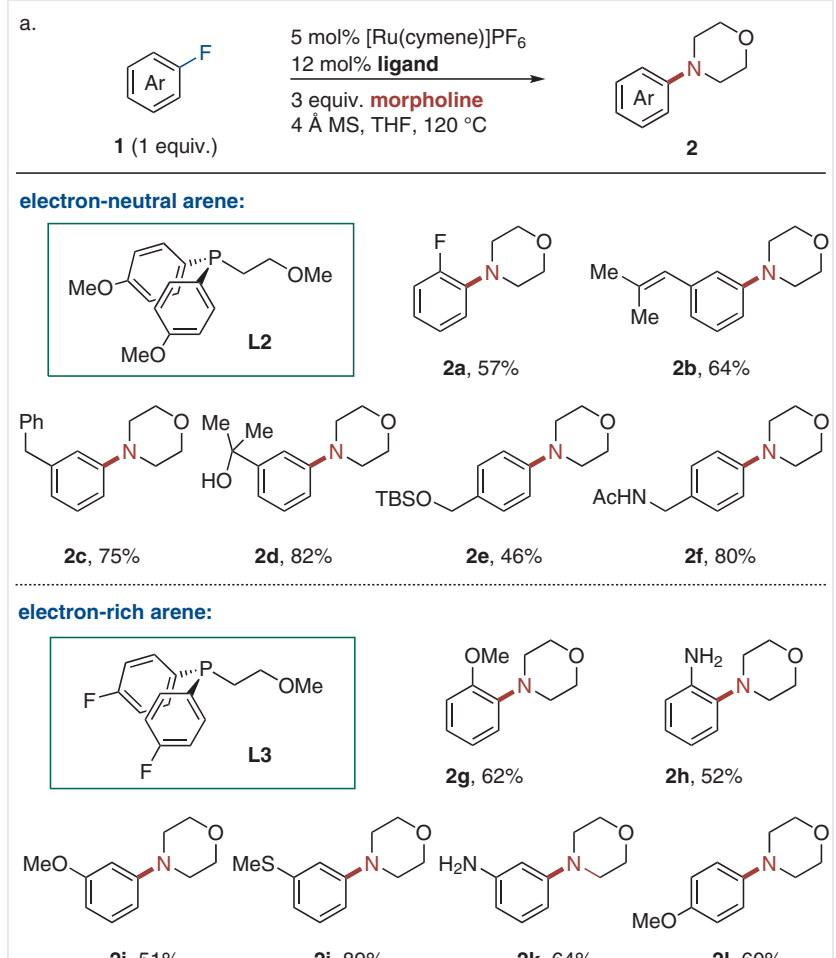

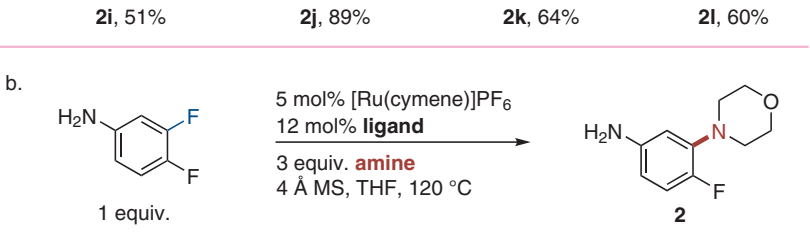

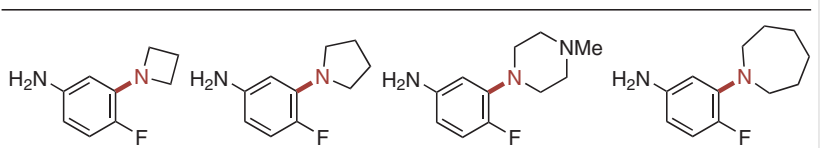
$2 \mathrm{~m}$, trace 2n, $50 \%$

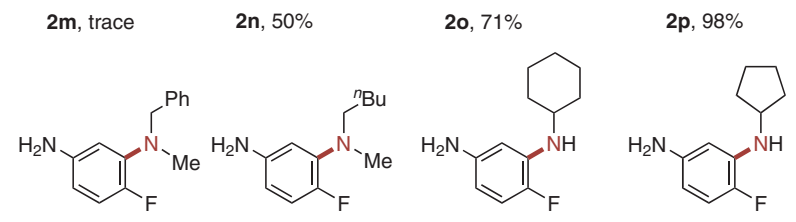

2p, $98 \%$

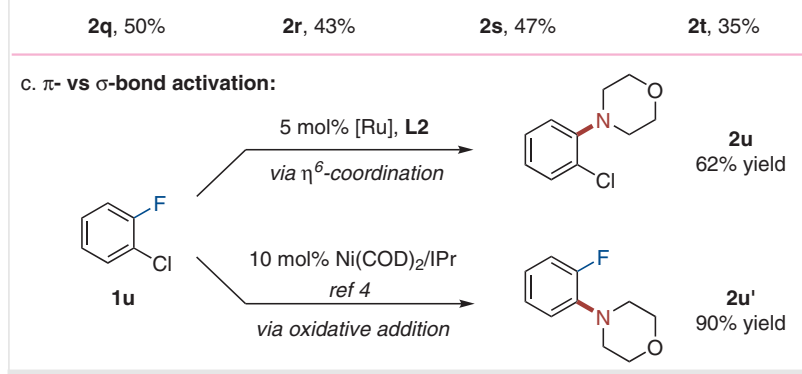

Scheme 5 Substrate scope 
hemilabile ligand. Further optimization of ligands by modifying substituted aryl groups and reaction conditions enhanced the yield to $95 \%$.

With the optimized amination conditions in hand, we then tested the substrate scope with morpholine (Scheme 5 , a). A broad range of electron-neutral arenes were compatible by using ligand L2 (2a-f), while ligand $\mathbf{L} 3$ bearing an electron-withdrawing group performed better on electronrich substrates than $\mathbf{L} \mathbf{2}(\mathbf{2 g}-\mathbf{1})$. Next, the scope of amine was examined with 3,4-difluoroaniline (Scheme 5, b). The 4membered amine azetidine was not tolerated under the conditions (2m). In comparison, 5-, 6-, and 7-membered cyclic amines were proved to be suitable nucleophiles (2np). Linear secondary and primary amines were also com-

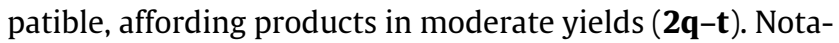
bly, a $\mathrm{C}-\mathrm{Cl}$ bond in $\mathbf{1} \mathbf{u}$, which is more reactive than a $\mathrm{C}-\mathrm{F}$ bond towards oxidative addition, survived under the conditions (Scheme 5, c). In comparison, such $\mathrm{C}-\mathrm{Cl}$ bond was exclusively converted into a $\mathrm{C}-\mathrm{N}$ bond by employing the $\mathrm{Ni}-$ IPr catalyst $\left(\mathbf{2} \mathbf{u}^{\prime}\right)$.

To gain insights into the reaction, we tested the nucleophilic substitution of the proposed intermediator $\eta^{6}$-complex 4, which formed on treatment of $\mathbf{3}$ with silver salt and ligand $\mathbf{L 1}$ in sequence (Scheme 6, a). According to the ${ }^{1} \mathrm{H}$ NMR and ${ }^{31} \mathrm{P}$ NMR spectra, we propose that the two hemilabile ligands on intermediate $\mathbf{5}$ are asymmetric: one chelates the Ru by the phosphine and oxygen simultaneously, while the other is monocoordinated. The fluorine peak of complex 4 disappeared immediately after adding morpholine, suggesting the amination proceeds fast even at room temperature. Then, after heating the reaction mixture at 60 ${ }^{\circ} \mathrm{C}$, the free aniline was observed. The above observations supported that the $S_{N} A r$ reaction proceeds on the $\eta^{6}$-arene complex. Furthermore, we compared the hemilabile ligand with diphenylbutylphosphine bearing a noncoordinating butyl chain in the arene dissociation step, and found that the weakly coordinating group facilitates arene dissociation. The hemilabile ligand $\mathbf{L 1}$ supported complex 7 released the aniline completely in a half hour, while complex 8 was much more stable (Scheme 6, b).

We have developed a Ru/hemilabile-ligand-catalyzed $\mathrm{S}_{\mathrm{N}} \mathrm{Ar}$ amination of aryl fluorides as the limiting reagents. A significant ligand enhancement was demonstrated by utilizing the phosphine ligand bearing a weakly coordinating side chain. Mechanistic studies revealed that the substitution proceeds on the TM $\eta^{6}$-complex, and the weakly coordinating group of the hemilabile ligand promotes product dissociation.

Given the mild reaction conditions, as well as the broad functional group tolerance, this protocol may attract attentions in organic synthesis, including late-stage C-F bond diversifications. Further studies into the mechanism and the extension to other catalytic reactions via $\eta^{6}$-coordination are currently ongoing.

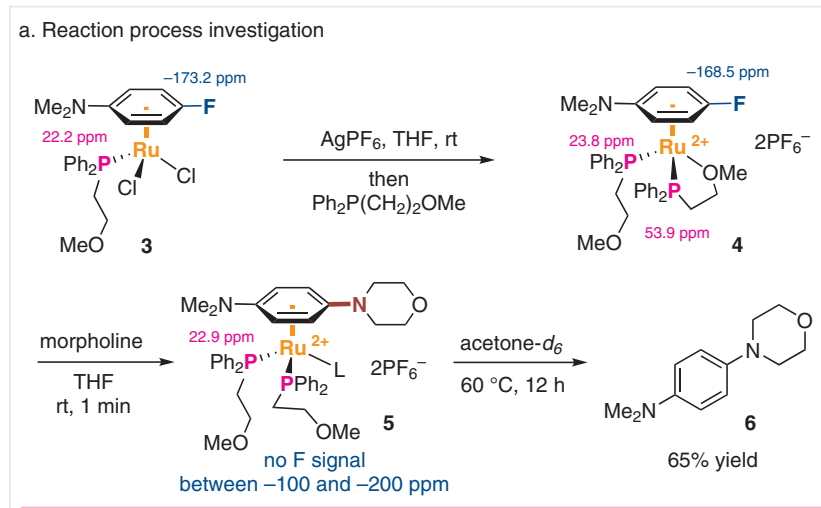

b. Arene dissociation test
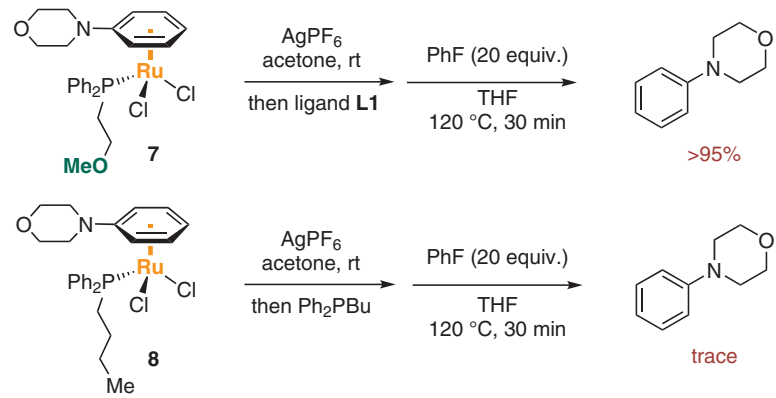

Scheme 6 Mechanistic study

\section{Funding Information}

This project was supported by Foundation of Westlake University and China Postdoctoral Science Foundation (2019M662118).

\section{References}

(1) For selected reviews, see: (a) Hartwig, J. F. Acc. Chem. Res. 1998, 31, 852. (b) Wolfe, J. P.; Wagaw, S.; Marcoux, J. F.; Buchwald, S. L. Acc. Chem. Res. 1998, 31, 805. (c) Bariwalab, J.; van der Eycken, E. Chem. Soc. Rev. 2013, 42, 9283. For selected examples, see: (d) Guram, A. S.; Rennels, R. A.; Buchwald, S. L. Angew. Chem., Int. Ed. Engl. 1995, 34, 1348. (e) Louie, J.; Hartwig, J. F. Tetrahedron Lett. 1995, 36, 3609.

(2) For selected reviews, see: (a) Monnier, F.; Taillefer, M. Top. Organomet. Chem. 2013, 46, 173. For selected examples, see: (b) Klapars, A.; Antilla, J. C.; Huang, X.; Buchwald, S. L. J. Am. Chem. Soc. 2001, 123, 7727. (c) Zhou, W.; Fan, M.; Yin, J.; Jiang, Y.; Ma, D. J. Am. Chem. Soc. 2015, 137, 11942.

(3) Kiso, Y.; Tamao, K.; Kumada, M. J. Organomet. Chem. 1973, 50, C12.

(4) Zhu, F.; Wang, Z.-X. Adv. Synth. Catal. 2013, 355, 3694.

(5) Harada, T.; Ueda, Y.; Iwai, T.; Sawamura, M. Chem. Commun. 2018, 54, 1718.

(6) Wang, Y.; Wei, C.; Tang, R.; Zhan, H.; Lin, J.; Liu, Z.; Tao, W.; Fang, Z. Org. Biomol. Chem. 2018, 16, 6191.

(7) For selected reviews of $S_{N} A r$ reaction, see: (a) Carey, F. A.; Sundberg, R. J. Advanced Organic Chemistry: Part A: Structure and Mechanisms, 4th ed.; Springer: New York, 2000, 589. (b) Terrier, F. Modern Nucleophilic Aromatic Substitution; Wiley- 
VCH: Weinheim, 2013, e-book ISBN. (c) Neumann, C. N.; Ritter, T. Acc. Chem. Res. 2017, 50, 2822. (d) Rohrbach, S.; Smith, A. J.; Pang, J. H.; Poole, D. L.; Tuttle, T.; Chiba, S.; Murphy, J. A. Angew. Chem. Int. Ed. 2019, 58, 16368.

(8) For selected reviews, see: (a) Pike, R. D.; Sweigart, D. A. Coord. Chem. Rev. 1999, 187, 183. (b) Pape, A. R.; Kaliappan, K. P.; Kündig, E. P. Chem. Rev. 2000, 100, 2917. (c) Pigge, F. C.; Coniglio, J. J. Curr. Org. Chem. 2001, 5, 757. (d) Semmelhack, M. F.; Chlenov, A. Top. Organomet. Chem. 2004, 7, 43. For selected recent examples, see: (e) Semmelhack, M. F.; Hilt, G.; Colley, J. H. Tetrahedron Lett. 1998, 39, 7683. (f) Kamikawa, K.; Kinoshita, S.; Furusyo, M.; Takemoto, S.; Matsuzaka, H.; Uemura, M. J. Org. Chem. 2007, 72, 3394. (g) Braun, W.; Calmuschi-Cula, B.; Englert, U.; Höfener, K.; Alberico, E.; Salzer, A. Eur. J. Org. Chem. 2008, 2065. (h) McGrew, G. I.; Temaismithi, J.; Carroll, P. J.; Walsh, P. J. Angew. Chem. Int. Ed. 2010, 49, 5541. (i) McGrew, G. I.; Stanciu, C.; Zhang, J.; Carroll, P. J.; Dreher, S. D.; Walsh, P. J. Angew. Chem. Int. Ed. 2012, 51, 11510. (j) Miller, A. J. M.; Kaminsky, W.; Goldberg, K. I. Organometallics 2014, 33, 1245. (k) Shirakawa, S.; Yamamoto, K.; Maruoka, K. Angew. Chem. Int. Ed. 2015, 54, 838. (l) D'Amato, E. M.; Neumann, C. N.; Ritter, T. Organometallics 2015, 34, 4626. (m) Mao, J.; Zhang, J.; Jiang, H.; Bellomo, A.; Zhang, M.; Gao, Z.; Dreher, S. D.; Walsh, P. J. Angew.
Chem. Int. Ed. 2016, 55, 2526. (n) Beyzavi, M. H.; Mandal, D.; Strebl, M. G.; Neumann, C. N.; D'Amato, E. M.; Chen, J.; Hooker, J. M.; Ritter, T. ACS Cent. Sci. 2017, 3, 944. (o) Pike, J. A.; Walton, J. W. Chem. Commun. 2017, 53, 9858.

(9) Fischer, E. O.; Hafner, W. Z. Naturforsch., B: Anorg. Chem., Org. Chem., Biochem. , Biophys., Biol. 1955, 10b, 665.

(10) For selected reviews, see: (a) Wilkinson, L. A.; Walton, J. W. Organomet. Chem. 2019, 42, 125. (b) Takemoto, S.; Matsuzaka, H. Tetrahedron Lett. 2018, 59, 697. For catalytic $S_{N} A r$ reactions via $\mathrm{n}^{6}$-coordination, see: (c) Houghton, R. P.; Voyle, M.; Price, R. J. Chem. Soc., Perkin Trans. 1 1984, 925. (d) Otsuka, M.; Endo, K.; Shibata, T. Chem. Commun. 2010, 46, 336. (e) Otsuka, M.; Yokoyama, H.; Endo, K.; Shibata, T. Synlett 2010, 2601. (f) Konovalov, A. I.; Gorbacheva, E. O.; Miloserdov, F. M.; Grushin, V. V. Chem. Commun. 2015, 51, 13527. (g) Walton, J. W.; Williams, J. M. J. Chem. Commun. 2015, 51, 2786.

(11) Mahaffy, C. A. L.; Pauson, P. L. J. Chem. Res., Synop. 1979, 126.

(12) (a) Semmelhack, M. F.; Seufert, W.; Keller, L. J. Organomet. Chem. 1982, 226, 183. (b) Kündig, E. P.; Kondratenko, M.; Romanens, P. Angew. Chem. Int. Ed. 1998, 37, 3146. (c) Semmelhack, M. F.; Chlenov, A.; Ho, D. M. J. Am. Chem. Soc. 2005, 127, 7759.

(13) Kang, Q.-K.; Lin, Y.; Li, Y.; Shi, H. J. Am. Chem. Soc. 2020, 142, 3706. 